\title{
Bronchodilators in bronchiolitis: Yes or no?
}

Acute respiratory infections (ARI) continue to be the main cause of disease in the first years of life, being bronchiolitis the most frequent entity. Because this problem repeats year after year, different public health strategies have been adopted by many countries to deal with it.

In Latin America, Chile was the first country to develop a specific program for ARI. It was based on identifying the clinical problem (i.e.: croup, pneumonia, obstructive bronchial syndrome -OBS-), assessing their severity, starting the appropriate treatment and, eventually, quickly refereeing to a secondary health center. ${ }^{1}$ In the case of OBS, the severity was assessed by a clinical score, and the initial treatment included salbutamol administered by metered-dose inhaler (MDI) with a valved holding chamber (VHC), and, eventually, oxygen if necessary. In this approach, patients with bronchiolitis are included as having a OBS. This strategy was very successful, being adopted by different countries of the region. The current Argentine, Chilean and Peruvian guidelines continue to focus the attention of infants with ARI and wheezing based on the concept of OBS and, therefore, including adrenergic bronchodilators via MDI as "therapeutic test".

The 2006 American Academy of Pediatrics (AAP) bronchiolitis guideline also considered the use of bronchodilators as a "therapeutic test". However, in its latest version (2014), it recommends not including adrenergic bronchodilators as a therapeutic option. ${ }^{2}$ The authors argue that, given the strong evidence of non-benefit and, that there is no well-established objective method to evaluate response to bronchodilators, they should not be routinely used in bronchiolitis. But, they recognize that "the population of children with bronchiolitis included in most studies limits the ability to make recommendations for all clinical scenarios".

We think that the 2014 AAP recommendation is questionable for the following reasons: a) All randomized studies carried out in bronchiolitis in which bronchodilators did not show significant improvement were performed using salbutamol by nebulization and not by MDI; ${ }^{3}$ the lower response to salbutamol administered via nebulization than via MDI with VHC is well known. ${ }^{4} \mathrm{~b}$ ) At least one randomized study showed that children younger than 1 year old with wheezing who received salbutamol by MDI with VHC had a better response (less likely to require hospitalization and greater improvement in clinical score) than those who received salbutamol by nebulization. ${ }^{5}$ c) Although most children with acute bronchiolitis will not develop asthma, there is a subset of patients younger than one year of age in whom bronchiolitis is the first manifestation of an asthmatic crisis, and therefore will benefit from a "therapeutic test" with salbutamol by MDI with VHC.

We also should consider that differences in the definition of bronchiolitis exists worldwide. For example, according to the Italian consensus, bronchiolitis is restricted to the first episode in a child less than 12 month of age, ${ }^{6}$ whereas the AAP guidelines do not limit it to the first year of life, and point out the presence of wheezing. ${ }^{2}$ Moreover, the British bronchiolitis guideline states that we should think of an early asthma attack rather than bronchiolitis if the episode occurs in children close to 12 months or older, who have persistent wheezing without crackles, episodes of recurrent wheezing or family history of asthma. ${ }^{7}$ This indicates that with the current definitions of "bronchiolitis" used around the world, we cannot be sure if we are dealing with an infant with just bronchiolitis or with one with their first episode of asthma, especially considering the high prevalence of this condition. Rather, it points to the fact that "bronchiolitis" is a heterogeneous disease in its clinical features, and depends on several factors such as the virus and its interaction with the airway microbiome, the immune response, the host genetics, and environmental factors. ${ }^{8,9}$ Therefore, it is very likely that the same therapeutic scheme should not be the most appropriate to treat all patients.

On the other hand, it should not be forgotten that the strategy used in Argentina focus in children with OBS up to 2 years old. Therefore, it is more likely that along with bronchiolitis, patients with asthma will be included in their first episode.

Regardless of this controversy arising from the available evidence, the experience in the management of this disease has left us some concepts:

1. Most patients with this condition contact the health system through primary care or emergency departments and, therefore, 
the strategies for their management must contemplate this reality.

2. It is important to categorize the severity of the patients with a clinical score for identifying those that can be safely managed as outpatient, those who need to be closely observed for some time, and those that demand immediate hospitalization. The clinical scores used in both Chile and Argentina are useful and correlates adequately with the ranges of SatO2 requiring intervention, ${ }^{10}$ and allows to evaluate the response to bronchodilator treatment.

3. Oxygen is the only treatment essential when there is hypoxemia.

4. The use of salbutamol as a "therapeutic test" may be beneficial, and should be used until new trials show conclusive evidence (i.e., randomized clinical studies with salbutamol via MDI with WHC vs. placebo), at least while national public health programs are based on the concept of OBS.

However, it is also important to emphasize that, with no response to salbutamol, treatment should be discontinued. The lack of courage to stop a bronchodilator treatment once proven ineffective, should not prevent some patients from receiving effective treatment, nor limiting the therapeutic resources of primary care physicians.

Fernando Ferrero, $M D, P h D$

Departamento de Medicina, Hospital General de Niños Pedro de Elizalde, Buenos Aires

José A. Castro-Rodríguez, $M D, P h D$ División de Pediatría, Escuela de Medicina, Pontificia Universidad Católica de Chile http:/ / dx.doi.org/10.5546/ aap.2018.eng.5

To cite: Ferrero F, Castro-Rodríguez JA. Bronchodilators in bronchiolitis: Yes or no? Arch Argent Pediatr 2018;116(1):5-6.

\section{REFERENCES}

1. Girardi G, AstudilloP, Zúñiga F. El programa IRA en Chile: hitos e historia. Rev Chil Pediatr 2001;72(4):292-300.

2. Ralston SL, Lieberthal AS, Meissner HC, et al. Clinical practice guideline: the diagnosis, management, and prevention of bronchiolitis. Pediatrics 2014;134(5):e1474502.

3. Castro-Rodriguez JA, Rodriguez-Martinez CE, SossaBriceño MP. Principal findings of systematic reviews for the management of acute bronchiolitis in children. Paediatr Respir Rev 2015;16(4):267-75.

4. Castro-Rodríguez JA, Rodrigo GJ. Beta-agonists through metered-dose inhaler with valved holding chamber versus nebulizer for acute exacerbation of wheezing or asthma in children under 5 years of age: a systematic review with meta-analysis. J Pediatr 2004;145(2):172-7.

5. Rubilar L, Castro-Rodriguez JA, Girardi G. Randomized trial of salbutamol via metered-dose inhaler with a spacer versus nebulizer for acute wheezing in children less than 2 years of age. Pediatr Pulmonol 2000;29(4):264-9.

6. Baraldi E, Lanari M, Manzoni P, etal.Inter-society consensus document on treatment and prevention of bronchiolitis in newborns and infants. Ital J Pediatr 2014;40:65.

7. National Institute for Health Care and Health Excellence. Bronchiolitis in children: diagnosis and management. NICE guideline [NG9]. London: NICE; 2015. [Acceso: 13 de octubre de2017]. Disponible en: https: / / www.nice.org. uk/guidance/ng9/

8. Dumas O, Mansbach JM, Jartti T, etal. A clustering approach to identify severe bronchiolitis profiles in children. Thorax 2016;71(8):712-8

9. Mansbach JM, Clark S, Teach SJ, et al. Children hospitalized with rhinovirus bronchiolitis have asthmalike characteristics. J Pediatr 2016;172:202-4.

10. Coarasa A, Giugno H, Cutri A, et al. Validación de una herramienta de predicción clínica simple para la evaluación dela gravedad en niños con síndrome bronquial obstructivo. Arch Argent Pediatr 2010;108(2):116-23. 